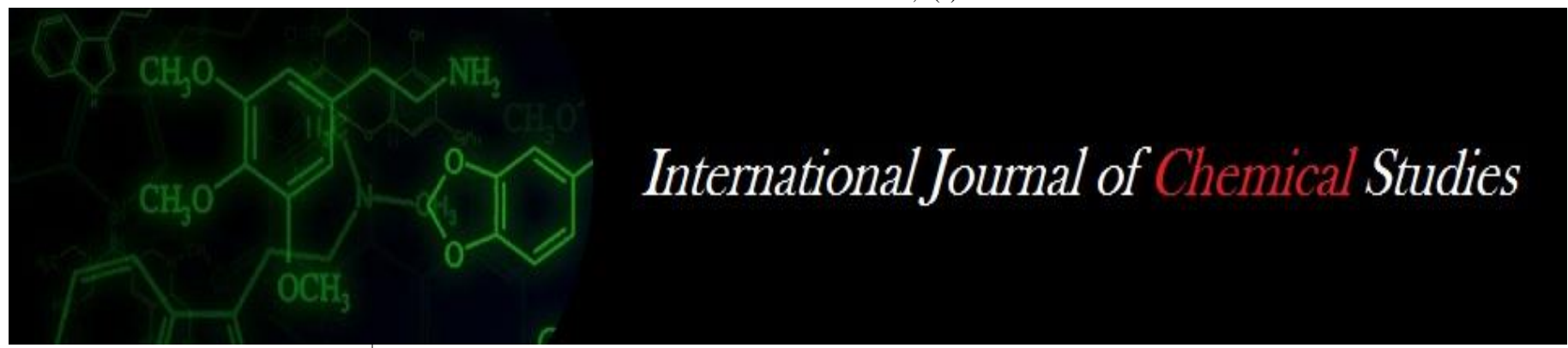

P-ISSN: 2349-8528

E-ISSN: 2321-4902

IJCS 2020; 8(1): 1240-1243

(C) 2020 IJCS

Received: 16-11-2019

Accepted: 18-12-2019

Sanika Joshi

Department of Plant Pathology, DBSKKV, Dapoli, Maharashtra, India

\section{PG Borkar}

Department of Plant Pathology, DBSKKV, Dapoli, Maharashtra, India

MS Joshi

Department of Plant Pathology, DBSKKV, Dapoli, Maharashtra, India

\section{Integrated management of anthracnose disease of bottle gourd}

\section{Sanika Joshi, PG Borkar and MS Joshi}

DOI: https://doi.org/10.22271/chemi.2020.v8.i1q.8421

\begin{abstract}
The anthracnose of bottle gourd incited by Colletotrichum lagenarium (Syn.C. orbiculare) is emerging as a major disease of bottle gourd in Konkan region. The fungal growth on solid medium was robust, compact with distinct zonation at specific distance. Initially the growth was olivaceous grey to green which gradually turned black with a pinkish tinge at maturity. Mycelium was septate, hyaline and branched about 2-5 $\mu \mathrm{m}$ in diameter. Conidia were hyaline, single celled measuring about $12-14$ X 4 $4.5 \mu \mathrm{m}$ and guttulate with single oil globule. The disease appeared in the form minute, irregular yellow spots on leaves. These spots enlarged into brown necrotic lesions surrounded by yellow halo. Under field conditions, Benomyl $(0.25 \%)$ was found to be the most effective fungicide with PDI of 28.00 per cent. The highest yield 19.43t/ha was recorded from Propiconazole $(0.1 \%)$ with highest ICBR of 0.63 . in case of integrated management of the disease, seed treatment with $T$. viride + three sprays with Propiconazole $(0.1 \%)$ was the best treatment in integrated management with the lowest disease intensity $(24.35 \%)$. The maximum yield (20.71t/ha) was recorded from seed treatment with $T$. viride + three sprays of Propiconazole $(0.1 \%)$ with ICBR of 0.80 .
\end{abstract}

Keywords: Bottlegourd, Colletotrichum lagenarium, robust, anthracnose

\section{Introduction}

Bottle gourd [Lagenaria siceraria (Mol.) Standal.], belonging to family Cucurbitaceae is fruit vegetable. The crop is considered to be a native of tropical Africa. Its cultivation dates back to at least 4000 years by the Egyptians. It is one of the chief culinary vegetables in many tropical and temperate regions around the world. Bottle gourd is also known as calabash, white flowered gourd, long melon, New Guinea bean and Tasmania bean in different parts of world. It is called as doodhi, ghiya or lauki in India. L. siceraria is cultivated in India, Japan, Sri Lanka, China and Thailand for its kitchen use. It has high medicinal value and hence used in some ayurvedic medicines. Being low in fat and cholesterol, and high in dietary fiber, it is recommended by dieticians.

Bottle gourd is a good source of vitamin- B- complex and ascorbic acid. It is rich in pectin and also contains various saponins, fatty oils and alcohols. It has a cooling effect on the human body and is also useful in prevention of constipation (Jemima and Prasadini, 2011) ${ }^{[6]}$. Bottle gourd fruit contains 96.3 per cent water, 2.8 per cent carbohydrate, 0.2 per cent protein, 0.5 per cent fat, 0.5 per cent mineral matter $(\mathrm{Ca}-0.02 \%, \mathrm{P}-0.01 \%$ and $\mathrm{Fe}-0.0007 \%)$, vitamin $\mathrm{A}$ in traces and vitamin B- 10 I.U. per 100g (Chauhan,2002). It is one of the low calorie vegetables (14 calories per $100 \mathrm{~g}$ ). Fresh gourds contains about $6 \mu \mathrm{g} / 100 \mathrm{~g}$ of folic acid that helps in reducing the defects in newborns.

Major bottle gourd growing states of India are U.P., Punjab, Gujarat, Assam, Meghalaya and Rajasthan. In India about 153 thousand ha area is under the cultivation of bottle gourd with an annual production of 2529 thousand MT and productivity of 16.52 tons per ha. Production wise, Bihar is the leading state (631.60 thousand tones) with a share of 25.70 per cent followed by Uttar Pradesh and Haryana (Anonymous, 2017) ${ }^{[1]}$.

In Konkan region this crop is basically grown on large scale in Raigad and Thane districts. Bottle gourd is one of the kitchen garden crops in Ratnagiri district.

Among fungal diseases, downy mildew, powdery mildew anthracnose, and Cercospora leaf spot are more prevalent in different bottle gourd growing areas. Among all these, anthracnose caused by Colletotrichum lagenarium (Pass) Ellis and Halsted is of major economic importance.
Corresponding Author:

Sanika Joshi

Department of Plant Pathology, DBSKKV, Dapoli, Maharashtra, India 
This disease was also recorded as one of the important diseases of bottle gourd in Konkan region of Maharashtra (Shinde, 1993) ${ }^{[8]}$. Hot and humid climate of the region is highly congenial for disease development. As the bottle gourd crop is commercially cultivated in North Konkan region for local and metropolitan markets, it is necessary to reduce the qualitative and quantitative losses due to anthracnose in strategic manner.

\section{Material and Methods}

The field experiments during Kharif 2018 were conducted at Central Experiment Station, Wakawali, Tal. Dapoli, Dist. Ratnagiri. During Rabi 2018-19, the experiments were conducted at the field of Department of Agronomy, College of Agriculture, Dapoli. The field experiment was conducted on variety Samrat during both the trials. The experiment was laid in randomized block design (RBD). The crop was observed carefully for initiation of disease. Three sprays of bio-agents, plant extract and fungicides were applied at an interval of 15 days starting from initiation of disease symptoms. A field trial was conducted to evaluate the effect of combination of treatments. The treatments included, T1Seed treatment with Trichoderma viride +Three sprays of Propiconazole $(0.1 \%)$, T2- Seed treatment with Trichoderma viride + Three sprays of Thiophanate Methyl $(0.1 \%)$, T3Seed treatment with Trichoderma viride + Three sprays of Sapindus trifoliatus (30\%), T4- Three sprays of Propiconazole $(0.1 \%)$, T5- Three sprays of Thiophanate Methyl $(0.1 \%)$, T6Three sprays of Sapindus trifoliatus $(30 \%)$ and T7-untreated (control).

Per cent Disease Index (PDI) was calculated by the formula:

$$
\mathrm{PDI}=\frac{\text { Sum of all numerical ratings }}{\text { No. of observations assessed } \mathrm{X} \text { Maximum rating }} \mathrm{X} 100
$$

The per cent disease control was calculated by using the formula given below:

$$
\mathrm{PDC}=\frac{\mathrm{PDI} \text { in control }-\mathrm{PDI} \text { in treatment }}{\mathrm{PDI} \text { in control }} \times 100
$$

\section{Yield}

Fruits were harvested at maturity and yields of net plot were recorded as $\mathrm{kg}$ per plot and later expressed in tonnes per ha. The per cent increase in yield over control was calculated by using formula given below:

$$
\begin{gathered}
\text { Yield increase } \\
\text { over control }(\%)
\end{gathered}=\frac{\text { Yield in treatment plot }- \text { Yield in control plot }}{\text { Yield in control plot }} \times 100
$$

To find out the most effective and economical treatment, the incremental cost: benefit ratio (ICBR) was worked out. Total monetary gain per treatment was worked out on the basis of selling rates of bottle gourd in the local market. The data obtained in all the experiments were statistically analyzed using SAS software.

\section{Results and Discussion}

An in vivo trial for management of bottle gourd anthracnose was conducted in Kharif and rabi seasons. The results from Kharif, 2018 (Table1) revealed that, 30 DAS i.e. one day before first spraying the intensity ranged between 17.33 to 18.00 per cent. The PDI for plot with seed treated of $T$. viride
(@ $25 \mathrm{~g} / \mathrm{kg}$ ) ranged from 15.06 to 15.60 per cent. The lowest (terminal) disease intensity $(23.22 \%)$ was recorded in the treatment $\mathrm{T}_{1}$ [Seed treatment with $T$. viride + three sprays with Propiconazole $(0.1 \%)]$ which was statistically superior over all treatments. This was followed treatment $\mathrm{T}_{2}$ [Seed treatment with $T$. viride + three sprays with Thiophenate methyl $(0.1 \%)$ ] with disease intensity of 24.26 per cent and $\mathrm{T}_{3}$ [Seed treatment with $T$. viride + three sprays with Sapindus trifoliatus $(30 \%)$ ] with 25 per cent. The sole treatments comprising three sprays of Propiconazole, Thiphanate Methyl and plant extract were statistically significant with each other and superior to control but less effective than combination treatments. The highest per cent disease control $(60.88 \%)$ was observed in treatment $\mathrm{T}_{1}$ [Seed treatment with $T$. viride + three sprays with Propiconazole $(0.1 \%)$ ].

In Rabi, 2018-19 (Table 2), the lowest disease intensity $(25.47 \%)$ was recorded in the treatment $\mathrm{T}_{1}$ [Seed treatment with $T$. viride + three sprays with Propiconazole $(0.1 \%)$ ] which was statistically superior over all treatments. This was followed treatment $\mathrm{T}_{2}$ [Seed treatment with $T$. viride + three sprays with Thiophenate methyl $(0.1 \%)]$ with disease intensity of 26.37 per cent and $\mathrm{T}_{3}$ [Seed treatment with $T$. viride + three sprays with Sapindus trifoliatus (30\%)] with disease intensity of 27.38 per cent. Among rest of the treatments, sole sprays of Propiconazole and Thiphanate methyl were at par with each other and statistically significant with three sprays of Sapindus trifoliatus. The highest percentage disease control over control by $50.55 \%$ was observed in treatment $\mathrm{T}_{1}$ [Seed treatment with $T$. viride + three sprays with Propiconazole $(0.1 \%)]$.

Pooled analysis (Table3) revealed that, the lowest disease intensity $(24.35 \%)$ was recorded in the treatment $\mathrm{T}_{1}$ [Seed treatment with $T$. viride + three sprays with Propiconazole $(0.1 \%)]$ which was statistically superior over all treatments. This was followed treatment $\mathrm{T}_{2}$ [Seed treatment with $T$. viride + three sprays with Thiophenate methyl $(0.1 \%)]$ with disease intensity of 25.32 per cent and $\mathrm{T}_{3}$ [Seed treatment with $T$. viride + three sprays with Sapindus trifoliatus (30\%)] with disease intensity of 26.19 per cent. In pooled analysis also, the treatments T4 and T5 were at par and significantly superior to T6 and T7. The highest percentage disease control over control by $56.07 \%$ was observed in treatment $\mathrm{T}_{1}$ [Seed treatment with $T$. viride + three sprays with Propiconazole $(0.25 \%)]$. It was noticed that, seed treatment (Thiram $-3 \mathrm{~g} / \mathrm{kg}$ of seed), green manuring (glyricidia leaves $-1.5 \mathrm{t} / \mathrm{ha}$ ) and foliar sprays of Propiconazole $(0.1 \%)$ was most effective against bitter gourd anthracnose (Jadhav et al., 2010) ${ }^{[5]}$. Propiconazole $(0.1 \%)$ caused a dramatic reduction in disease incidence of chilli anthracnose by $70 \%$ (Gopinath et al., 2006) ${ }^{[4]}$. Dubale (2018) ${ }^{[3]}$ reported that, minimum disease severity of turmeric anthracnose (11.88\%) was observed in plot sprayed with Propiconazole $25 \%$ EC $(0.1 \%)$.

The pooled analysis from table 4 revealed that, the maximum yield $(20.71 \mathrm{t} / \mathrm{ha})$ was recorded from $\mathrm{T}_{1}$ [Seed treatment with T. viride + three sprays of Propiconazole $(0.1 \%)$ ] with $40.22 \%$ increase over control. It was followed by $\mathrm{T}_{2}$ [seed treatment with $T$. viride +3 sprays of Thiophanate methyl $(0.1 \%)-20.52 / \mathrm{ha}$ ] with 38.93 per cent increase over control. The treatment $\mathrm{T}_{4}$ [Three sprays of Propionazole $(0.1 \%)$ ] recorded 19.43 t/ha with 31.19 per cent increase over control was at par with $\mathrm{T}_{3}$ [Seed treatment with $T$. viride + three sprays with Sapindus trifoliatus (30\%)] which recorded yield of $19.34 \mathrm{t} /$ ha with $31.01 \%$ increase over control and $\mathrm{T}_{5}$ [Three sprays with Thiophanate methyl $(0.1 \%)$ recorded yield of $19.31 \mathrm{t} /$ ha with $30.74 \%$ increase over control. Least effective 
treatment was $\mathrm{T}_{6}$ [three sprays with Sapindus trifoliatus $(30 \%)$ ] with yield of $16.78 \mathrm{t} / \mathrm{ha}$ and 13.68 per cent increase over control. The yield recorded from control plot was 14.81t/ha. Potphode (2011) ${ }^{[7]}$ reported that, maximum yield $(48.61 \mathrm{q} / \mathrm{ha})$ was recorded from the treatment including seed dressing with Tricyclazole + three sprays of Difenconazole against anthracnose of bitter gourd. The economics of the trial conducted (pooled analysis) (Table 5) in both the seasons revealed that, the treatment $\mathrm{T}_{1}$ [Seed treatment with $T$. viride+
Three sprays of Propiconazole $(0.1 \%)]$ was found most effective with highest gross returns (Rs. 5,63,519/-) with additional income of Rs. 1,60,539/- and highest ICBR (0.80). An additional income of Rs.1, 55,369/- was recorded from $\mathrm{T}_{2}$ with ICBR of 0.77 . The treatments $\mathrm{T}_{5}$ and $\mathrm{T}_{3}$ recorded same ICBR (0.61). Least additional returns (Rs. 53,604/-) were recorded due to three sprays each of soapnut with ICBR of 0.26 .

Table1: Integrated Management of anthracnose with bio-agents, chemical fungicides and plant extract (Kharif, 2018)

\begin{tabular}{|c|c|c|c|c|c|c|}
\hline \multirow[b]{2}{*}{$\begin{array}{l}\text { Tr. } \\
\text { No. }\end{array}$} & \multirow[b]{2}{*}{ Treatment } & \multicolumn{4}{|c|}{ Per cent Disease Intensity (PDI) } & \multirow{2}{*}{$\begin{array}{l}\text { Per cent disease } \\
\text { control compared } \\
\text { to untreated }\end{array}$} \\
\hline & & \begin{tabular}{|c|} 
Before Spray \\
(30 DAS)
\end{tabular} & $\begin{array}{l}\text { First spray } \\
\text { (45 DAS) }\end{array}$ & $\begin{array}{c}\text { Second spray } \\
(60 \text { DAS })\end{array}$ & $\begin{array}{c}\text { Third spray } \\
\text { (75 DAS) }\end{array}$ & \\
\hline $\mathrm{T}_{1}$ & $\begin{array}{c}\text { Seed treatment with Trichoderma viride }+ \text { Three sprays of } \\
\text { Propiconazole }(0.1 \%)\end{array}$ & $\begin{array}{c}15.06 \\
(22.83) \\
\end{array}$ & $\begin{array}{c}18.57 \\
(25.53)\end{array}$ & \begin{tabular}{c|}
21.37 \\
$(27.54)$ \\
\end{tabular} & \begin{tabular}{|c|}
23.22 \\
$(28.80)$
\end{tabular} & 60.88 \\
\hline $\mathrm{T}_{2}$ & $\begin{array}{c}\text { Seed treatment with Trichoderma viride + Three sprays of } \\
\text { Thiophanate Methyl }(0.1 \%)\end{array}$ & $\begin{array}{l}15.77 \\
(23.40)\end{array}$ & $\begin{array}{l}19.38 \\
(26.12)\end{array}$ & $\begin{array}{c}22.17 \\
(28.09)\end{array}$ & $\begin{array}{l}24.26 \\
(29.51)\end{array}$ & 59.13 \\
\hline $\mathrm{T}_{3}$ & $\begin{array}{c}\text { Seed treatment with Trichoderma viride }+ \text { Three sprays of } \\
\text { Sapindus trifoliatus }(30 \%)\end{array}$ & $\begin{array}{l}15.60 \\
(23.26)\end{array}$ & $\begin{array}{c}19.05 \\
(25.88)\end{array}$ & $\begin{array}{c}22.73 \\
(28.47)\end{array}$ & $\begin{array}{c}25.00 \\
(30.00)\end{array}$ & 57.89 \\
\hline $\mathrm{T}_{4}$ & Three sprays ofPropiconazole $(0.1 \%)$ & $\begin{array}{c}17.33 \\
(24.60) \\
\end{array}$ & $\begin{array}{c}21.06 \\
(27.32) \\
\end{array}$ & $\begin{array}{c}25.72 \\
(30.48)\end{array}$ & $\begin{array}{c}26.79 \\
(31.17)\end{array}$ & 54.87 \\
\hline $\mathrm{T}_{5}$ & Three sprays of Thiophanate Methyl $(0.1 \%)$ & $\begin{array}{c}17.68 \\
(24.86)\end{array}$ & $\begin{array}{c}22.13 \\
(28.06) \\
\end{array}$ & $\begin{array}{c}25.52 \\
(30.34)\end{array}$ & $\begin{array}{c}27.15 \\
(31.40)\end{array}$ & 54.26 \\
\hline $\mathrm{T}_{6}$ & Three sprays of Sapindus trifoliatus (30\%) & $\begin{array}{c}17.58 \\
(24.79) \\
\end{array}$ & $\begin{array}{c}28.66 \\
(32.37) \\
\end{array}$ & $\begin{array}{c}36.84 \\
(37.37) \\
\end{array}$ & $\begin{array}{c}50.22 \\
(45.13) \\
\end{array}$ & 15.41 \\
\hline $\mathrm{T}_{7}$ & Control & $\begin{array}{l}18.00 \\
(25.10)\end{array}$ & $\begin{array}{c}30.86 \\
(33.75)\end{array}$ & $\begin{array}{c}44.81 \\
(42.02)\end{array}$ & $\begin{array}{c}59.37 \\
(50.40)\end{array}$ & - \\
\hline & S. Em \pm & 0.14 & 0.18 & 0.13 & 0.16 & - \\
\hline & C. $\mathrm{D}(\mathrm{P}=0.05)$ & 0.43 & 0.56 & 0.39 & 0.50 & - \\
\hline
\end{tabular}

Table 2: Integrated Management of anthracnose with bio-agents, chemical fungicides and plant extract (Rabi, 2018-19)

\begin{tabular}{|c|c|c|c|c|c|c|}
\hline \multirow{2}{*}{$\begin{array}{l}\text { Tr. } \\
\text { No. }\end{array}$} & \multirow[b]{2}{*}{ Treatment } & \multicolumn{4}{|c|}{\begin{tabular}{|c|} 
Per cent Disease Intensity (PDI) \\
\end{tabular}} & \multirow{2}{*}{$\begin{array}{l}\text { Per cent disease } \\
\text { control compared } \\
\text { to untreated }\end{array}$} \\
\hline & & $\begin{array}{c}\text { Before Spray } \\
\text { (30 DAS) }\end{array}$ & $\begin{array}{l}\text { First spray } \\
(45 \text { DAS })\end{array}$ & $\begin{array}{c}\text { Second spray } \\
(60 \text { DAS })\end{array}$ & $\begin{array}{c}\text { Third spray } \\
\text { (75 DAS) }\end{array}$ & \\
\hline $\mathrm{T}_{1}$ & $\begin{array}{c}\text { Seed treatment with Trichoderma viride }+ \text { Three sprays of } \\
\text { Propiconazole }(0.1 \%)\end{array}$ & $\begin{array}{c}17.68 \\
(24.86)\end{array}$ & $\begin{array}{c}19.45 \\
(26.17)\end{array}$ & \begin{tabular}{c|}
22.48 \\
$(28.30)$ \\
\end{tabular} & \begin{tabular}{|c|}
25.47 \\
$(30.31)$ \\
\end{tabular} & 50.55 \\
\hline $\mathrm{T}_{2}$ & $\begin{array}{c}\text { Seed treatment with Trichoderma viride }+ \text { Three sprays of } \\
\text { Thiophanate Methyl }(0.1 \%)\end{array}$ & $\begin{array}{l}18.28 \\
(25.31)\end{array}$ & $\begin{array}{c}20.37 \\
(26.83)\end{array}$ & $\begin{array}{c}23.55 \\
(29.03)\end{array}$ & $\begin{array}{c}26.37 \\
(30.90)\end{array}$ & 48.80 \\
\hline $\mathrm{T}_{3}$ & $\begin{array}{c}\text { Seed treatment with Trichoderma viride }+ \text { Three sprays of } \\
\text { Sapindus trifoliatus }(30 \%)\end{array}$ & $\begin{array}{c}18.49 \\
(25.46) \\
\end{array}$ & $\begin{array}{c}20.91 \\
(27.21) \\
\end{array}$ & $\begin{array}{c}24.69 \\
(29.79) \\
\end{array}$ & $\begin{array}{c}27.38 \\
(31.55) \\
\end{array}$ & 46.84 \\
\hline $\mathrm{T}_{4}$ & Three sprays of Propiconazole $(0.25 \%)$ & $\begin{array}{c}20.58 \\
(26.98)\end{array}$ & $\begin{array}{c}21.92 \\
(27.92)\end{array}$ & $\begin{array}{c}26.56 \\
(31.02)\end{array}$ & $\begin{array}{c}28.55 \\
(32.30)\end{array}$ & 44.57 \\
\hline $\mathrm{T}_{5}$ & Three sprays of Thiophanate Methyl $(0.1 \%)$ & $\begin{array}{l}21.78 \\
(27.82)\end{array}$ & $\begin{array}{c}23.95 \\
(29.29) \\
\end{array}$ & $\begin{array}{c}27.80 \\
(31.82) \\
\end{array}$ & $\begin{array}{c}28.95 \\
(32.55) \\
\end{array}$ & 43.79 \\
\hline $\mathrm{T}_{6}$ & Three sprays of Sapindus trifoliatus (30\%) & $\begin{array}{c}21.79 \\
(27.83)\end{array}$ & $\begin{array}{c}28.32 \\
(32.15)\end{array}$ & $\begin{array}{c}36.21 \\
(36.99)\end{array}$ & $\begin{array}{c}41.18 \\
(39.92)\end{array}$ & 20.05 \\
\hline $\mathrm{T}_{7}$ & Control & $\begin{array}{c}22.32 \\
(28.19)\end{array}$ & $\begin{array}{c}32.48 \\
(34.75)\end{array}$ & $\begin{array}{c}40.74 \\
(39.66)\end{array}$ & $\begin{array}{c}51.51 \\
(45.87)\end{array}$ & - \\
\hline & S. Em \pm & 0.17 & 0.20 & 0.16 & 0.20 & - \\
\hline & C. $\mathrm{D}(\mathrm{P}=0.05)$ & 0.54 & 0.63 & 0.49 & 0.63 & - \\
\hline
\end{tabular}

Table 3: Integrated Management of anthracnose with bio-agents, chemical fungicides and plant extract (Pooled)

\begin{tabular}{|c|c|c|c|c|c|c|}
\hline \multirow{2}{*}{$\begin{array}{l}\text { Tr. } \\
\text { No. }\end{array}$} & \multirow[b]{2}{*}{ Treatment } & \multicolumn{4}{|c|}{ Per cent Disease Intensity (PDI) } & \multirow{2}{*}{$\begin{array}{c}\text { Per cent disease } \\
\text { control compared } \\
\text { to untreated }\end{array}$} \\
\hline & & $\begin{array}{l}\text { Before Spray } \\
\text { (30 DAS) }\end{array}$ & $\begin{array}{l}\text { First spray } \\
(45 \text { DAS })\end{array}$ & $\begin{array}{c}\text { Second spray } \\
(60 \text { DAS })\end{array}$ & $\begin{array}{l}\text { Third spray } \\
\text { (75 DAS) }\end{array}$ & \\
\hline $\mathrm{T}_{1}$ & $\begin{array}{c}\text { Seed treatment with Trichoderma viride }+ \text { Three sprays of } \\
\text { Propiconazole }(0.1 \%)\end{array}$ & $\begin{array}{c}16.37 \\
(23.86) \\
\end{array}$ & $\begin{array}{c}19.01 \\
(25.85)\end{array}$ & \begin{tabular}{|c|}
21.93 \\
$(27.92)$ \\
\end{tabular} & $\begin{array}{c}24.35 \\
(29.57) \\
\end{array}$ & 56.07 \\
\hline $\mathrm{T}_{2}$ & $\begin{array}{c}\text { Seed treatment with Trichoderma viride }+ \text { Three sprays of } \\
\text { Thiophanate Methyl }(0.1 \%)\end{array}$ & $\begin{array}{c}17.03 \\
(24.37)\end{array}$ & $\begin{array}{c}19.88 \\
(26.48)\end{array}$ & $\begin{array}{c}22.86 \\
(28.56)\end{array}$ & $\begin{array}{c}25.32 \\
(30.21)\end{array}$ & 54.32 \\
\hline $\mathrm{T}_{3}$ & $\begin{array}{c}\text { Seed treatment with Trichoderma viride + Three sprays of } \\
\text { Sapindus trifoliatus }(30 \%)\end{array}$ & $\begin{array}{c}17.05 \\
(24.39)\end{array}$ & $\begin{array}{l}19.98 \\
(26.55)\end{array}$ & $\begin{array}{c}23.71 \\
(29.14)\end{array}$ & $\begin{array}{c}26.19 \\
(30.78)\end{array}$ & 52.75 \\
\hline $\mathrm{T}_{4}$ & Three sprays of Propiconazole $(0.1 \%)$ & $\begin{array}{c}18.96 \\
(25.81) \\
\end{array}$ & $\begin{array}{c}21.49 \\
(27.62)\end{array}$ & $\begin{array}{c}26.15 \\
(30.75) \\
\end{array}$ & $\begin{array}{c}27.67 \\
(31.74)\end{array}$ & 50.09 \\
\hline $\mathrm{T}_{5}$ & Three sprays of Thiophanate Methyl $(0.1 \%)$ & $\begin{array}{c}19.73 \\
(26.37)\end{array}$ & $\begin{array}{c}23.04 \\
(28.68)\end{array}$ & $\begin{array}{c}26.66 \\
(31.09)\end{array}$ & $\begin{array}{c}28.05 \\
(31.98)\end{array}$ & 49.40 \\
\hline $\mathrm{T}_{6}$ & Three sprays of Sapindus trifoliatus (30\%) & $\begin{array}{c}19.69 \\
(26.34)\end{array}$ & $\begin{array}{c}28.49 \\
(32.26)\end{array}$ & $\begin{array}{c}36.53 \\
(37.18)\end{array}$ & $\begin{array}{c}45.70 \\
(42.53)\end{array}$ & 17.56 \\
\hline
\end{tabular}




\begin{tabular}{|c|c|c|c|c|c|c|}
\hline \multirow{2}{*}{$\mathrm{T}_{7}$} & Control & $\begin{array}{c}20.16 \\
(26.68)\end{array}$ & $\begin{array}{c}31.67 \\
(34.25)\end{array}$ & $\begin{array}{c}42.77 \\
(40.84)\end{array}$ & $\begin{array}{c}55.44 \\
(48.12)\end{array}$ & - \\
\hline & S. Em \pm & 0.10 & 0.15 & 0.10 & 0.14 & - \\
\hline & C. D $(\mathrm{P}=0.05)$ & 0.30 & 0.47 & 0.30 & 0.45 & - \\
\hline
\end{tabular}

Table 4: Effect of Integrated Management on yield of bottle gourd.

\begin{tabular}{|c|c|c|c|c|c|c|c|}
\hline \multirow[t]{2}{*}{ Tr. No. } & \multirow[t]{2}{*}{ Treatment } & \multicolumn{2}{|c|}{ Kharif,2018 } & \multicolumn{2}{|c|}{ Rabi, 2018-19 } & \multicolumn{2}{|c|}{ Pooled } \\
\hline & & $\begin{array}{c}* \text { Yield } \\
(\mathbf{t} / \text { ha. })\end{array}$ & \begin{tabular}{|c|}
$\%$ increase \\
over control
\end{tabular} & $\begin{array}{l}* \text { Yield } \\
\text { (t/ha.) }\end{array}$ & \begin{tabular}{|c|}
$\%$ increase \\
over control
\end{tabular} & $\begin{array}{l}\text { *Yield } \\
\text { (t/ha.) }\end{array}$ & $\begin{array}{c}\% \text { increase } \\
\text { over control }\end{array}$ \\
\hline $\mathrm{T}_{1}$ & $\begin{array}{l}\text { Seed treatment with Trichoderma viride }+ \text { Three sprays of Propiconazole } \\
(0.1 \%)\end{array}$ & 21.12 & 41.00 & 20.24 & 39.01 & 20.71 & 40.22 \\
\hline $\mathrm{T}_{2}$ & $\begin{array}{c}\text { Seed treatment with Trichoderma viride + Three sprays of Thiophanate } \\
\text { Methyl }(0.1 \%)\end{array}$ & 20.58 & 37.13 & 20.43 & 40.32 & 20.52 & 38.93 \\
\hline $\mathrm{T}_{3}$ & $\begin{array}{l}\text { Seed treatment with Trichoderma viride + Three sprays of Sapindus } \\
\text { trifoliatus }(30 \%)\end{array}$ & 19.06 & 27.13 & 19.63 & 34.82 & 19.34 & 31.01 \\
\hline $\mathrm{T}_{4}$ & Three sprays of Propiconazole $(0.1 \%)$ & 19.91 & 32.73 & 18.93 & 30.01 & 19.43 & 31.19 \\
\hline $\mathrm{T}_{5}$ & Three sprays of Thiophanate Methyl $(0.1 \%)$ & 19.69 & 30.80 & 19 & 30.49 & 19.31 & 30.74 \\
\hline $\mathrm{T}_{6}$ & Three sprays of Sapindus trifoliatus (30\%) & 16.39 & 9.13 & 17.20 & 18.13 & 16.78 & 13.68 \\
\hline $\mathrm{T}_{7}$ & Control & 15.00 & & 14.56 & & 14.81 & - \\
\hline & S.Em \pm & 0.54 & - & 0.28 & - & 0.29 & - \\
\hline & C.D $(\mathrm{P}=0.05)$ & 1.66 & - & 0.87 & - & 0.88 & - \\
\hline
\end{tabular}

* Mean of three replications

Table 5: Economics of fungicides, bio-agent and botanical in integrated management of bottle gourd anthracnose (Pooled)

\begin{tabular}{|c|c|c|c|c|c|c|c|c|c|c|}
\hline \multirow[b]{2}{*}{$\begin{array}{l}\text { Tr. } \\
\text { No. }\end{array}$} & \multirow[b]{2}{*}{ Treatment } & \multirow[b]{2}{*}{$\begin{array}{c}\text { Basic } \\
\text { input cost } \\
\quad(\text { Rs. })\end{array}$} & \multicolumn{2}{|c|}{ Additional cost (Rs.) } & \multirow{2}{*}{$\begin{array}{l}\text { Total } \\
\text { input } \\
\text { cost } \\
\text { (Rs.) }\end{array}$} & \multirow[b]{2}{*}{$\begin{array}{l}\text { *Yield } \\
(\mathrm{t} / \mathrm{ha} \text {. })\end{array}$} & \multirow[b]{2}{*}{$\begin{array}{c}* * \text { Gross } \\
\text { returns } \\
\text { (Rs.) }\end{array}$} & \multirow{2}{*}{$\begin{array}{l}\text { Increase in } \\
\text { yield over } \\
\text { control } \\
\text { (T/ha.) }\end{array}$} & \multirow{2}{*}{$\begin{array}{l}\text { Additional } \\
\text { returns due } \\
\text { to treatment } \\
\text { (Rs.) }\end{array}$} & \multirow[b]{2}{*}{ ICBR } \\
\hline & & & \begin{tabular}{|c|} 
Fungicide/ \\
bioagent/ organical \\
/phyto Extract
\end{tabular} & $\begin{array}{c}\text { Labour } \\
\text { cost } \\
\text { (Rs.) }\end{array}$ & & & & & & \\
\hline $\mathrm{T}_{1}$ & $\begin{array}{c}\text { Seed treatment with Trichoderma } \\
\text { viride }+ \text { Three sprays of } \\
\text { Propiconazole }(0.1 \%) \\
\end{array}$ & $1,95,155$ & 1,850 & 1800 & $1,98,805$ & 20.71 & $5,63,519$ & 5.90 & $1,60,539$ & 0.80 \\
\hline $\mathrm{T}_{2}$ & $\begin{array}{c}\text { Seed treatment with Trichoderma } \\
\text { viride + Three sprays of } \\
\text { Thiophanate Methyl }(0.1 \%)\end{array}$ & $1,95,155$ & 2,525 & 1800 & $1,99,480$ & 20.52 & $5,58,349$ & 5.71 & $1,55,369$ & 0.77 \\
\hline $\mathrm{T}_{3}$ & $\begin{array}{c}\begin{array}{c}\text { Seed treatment with Trichoderma } \\
\text { viride }+ \text { Three sprays of Sapindus } \\
\text { trifoliatus }(30 \%)\end{array} \\
\end{array}$ & $1,95,155$ & 3,275 & 1800 & $2,00,230$ & 19.34 & $5,26,241$ & 4.53 & $1,23,261$ & 0.61 \\
\hline $\mathrm{T}_{4}$ & $\begin{array}{c}\text { Three sprays of Propiconazole } \\
(0.1 \%)\end{array}$ & $1,95,155$ & 1,575 & 1800 & $1,98,530$ & 19.43 & $5,28,690$ & 4.62 & $1,25,710$ & 0.63 \\
\hline $\mathrm{T}_{5}$ & $\begin{array}{c}\text { Three sprays of Thiophanate } \\
\text { Methyl }(0.1 \%)\end{array}$ & $1,95,155$ & 2,250 & 1800 & $1,99,205$ & 19.31 & $5,25,425$ & 4.5 & $1,22,445$ & 0.61 \\
\hline $\mathrm{T}_{6}$ & $\begin{array}{c}\text { Three sprays of Sapindus } \\
\text { trifoliatus }(30 \%)\end{array}$ & $1,95,155$ & 3,000 & 1800 & $1,99,955$ & 16.78 & $4,56,584$ & 1.97 & 53,604 & 0.26 \\
\hline $\mathrm{T}_{7}$ & Control & $1,95,155$ & - & - & $1,95,155$ & 14.81 & & - & - & - \\
\hline
\end{tabular}

*Mean of three replications **Selling rates of bottle gourd@Rs.27210/t

\section{Conclusion}

The disease can be effectively managed by integration of the treatments comprising combination of bio-agent and fungicides. Seed treatment with Trichoderma viride followed by three sprays of Propiconazole $(0.1 \%)$ commencing from 30 DAS followed by two more sprays at 15 days interval was the best economical treatment in terms disease control and yield maximization.

\section{References}

1. Anonymous. National Horticultural Board Database, India, 2017.

2. Chauhan RS. Studies on anthracnose disease of bottle gourd caused by Colletotrichum lagenarium (PASS.) ELL. And HALST. Ph.D. (Agri.).Thesis submitted to Haryana Agricultural University, Hisar, 2002.

3. Dubale YD. Studies on management of Turmeric leaf spot caused by Colletotrichum spp. A MSc. Thesis submitted to Dr. B.S.K.K.V. Dapoli. (M.S), 2018.

4. Gopinath K, Radhakrishnana NV, Jayraj J. Effect of propiconazole and difenoconazole on the control of anthracnose of chilli fruits caused by Colletotrichum capsici. Crop Protection. 2006; 25(9):1024-1031.

5. Jadhav DK, Fugro PA, Kadam JJ. Management of anthracnose and root-knot nematode complex and evaluation of resistance source in bitter gourd. Journal of Plant Disease Sciences. 2010; 5(2):238-241.

6. Jemima MB, Prasadini. Nutrient composition and antioxidant activity of raw and processed bottle gourd varieties. Food Science. 2011; 36:3122-3124.

7. Potphode PD. Epidemiology and management of anthracnose of bitter gourd (Momordica charantia L.) incited by Colletotrichum lagenarium (Pass.) Ell. And Halst. A PhD. thesis submitted to DBSKKV, Dapoli (M.S.), 2011.

8. Shinde NS. Studies on anthracnose disease of cucurbits incited by Colletotrichum lagenarium (Pass.) Ell. And Halst. A M.Sc. thesis submitted to DBSKKV, Dapoli (M.S.), 1993. 